Rev. Bras. Saúde Prod. Anim., Salvador, v.15, n.4, p.827-834 out./dez., 2014 http://www.rbspa.ufba.br ISSN 15199940

\title{
Composição mineral da cana-de-açúcar in natura hidrolisada com cal virgem (CaO)
}

\author{
Mineral composition of cane sugar "in natura" hydrolyzed with quicklime (CaO)
}

\author{
DOMINGUES, Felipe Nogueira ${ }^{1 *}$; OLIVEIRA, Mauro Dal Secco de ${ }^{2}$; MOTA, Diego \\ Azevedo $^{3}$; OLIVEIRA, Raimundo Parente de ${ }^{4}$; SANTOS, Juliana dos ${ }^{5}$; MIRANDA, \\ Augusto Sousa ${ }^{6}$, GODOY, Bruno Spacek ${ }^{6}$
}

\author{
${ }^{1}$ Universidade Federal do Pará, Faculdade de Medicina Veterinária, Castanhal, Pará. \\ ${ }^{2}$ Universidade Estadual Paulista, Faculdade de Ciências Agrárias e Veterinárias, Departamento de \\ Zootecnia, Jaboticabal, São Paulo, Brasil. \\ ${ }^{3}$ Universidade Federal da Fronteira Sul, Erechim, Rio Grande do Sul, Brasil \\ ${ }^{4}$ Engenheiro Agrônomo, Belém, Pará, Brasil. \\ ${ }^{5}$ Instituto Federal do Rio Grande do Sul, Campus Sertão, Rio Grande do Sul, Brasil. \\ ${ }^{6}$ Universidade Federal do Pará, Programa de Pós-Graduação em Ciência Animal, Belém, Pará, Brasil. \\ *Endereço para correspondência: felipend@gmail.com
}

\section{RESUMO}

Objetivou-se avaliar a composição mineral da cana-de-açúcar in natura hidrolisada com doses crescentes de cal virgem e diferentes tempos de exposição ao ar. As doses de cal virgem $(\mathrm{CaO})$ utilizadas foram 0,0 (controle); 0,$5 ; 1,0 ; 1,5 \mathrm{e}$ $2,0 \%$ com base na matéria natural da cana e os tempos de exposição ao ar 0; 24; 48; 72 e 96 horas. A composição mineral foi avaliada através das concentrações cálcio $(\mathrm{Ca})$, fósforo $(\mathrm{P})$, potássio $(\mathrm{K})$, magnésio $(\mathrm{Mg})$; zinco $(\mathrm{Zn})$, cobre $(\mathrm{Cu})$, manganês $(\mathrm{Mn})$, cromo $(\mathrm{Cr})$, chumbo $(\mathrm{Pb}) \mathrm{e}$ cádmio $(\mathrm{Cd})$. Utilizou-se o delineamento inteiramente casualizado. Os dados foram analisados utilizando uma análise de variância com medidas repetidas, para a comparação das médias foi utilizado o teste de Tukey ao nível de $5 \%$ de probabilidade. A variação no teor de $\mathrm{Ca}$ $(\mathrm{P}<0,05)$ ocasionou alteração na relação $\mathrm{Ca}: \mathrm{P}$ da cana-de-açúcar hidrolisada, chegando a relação de $36,46: 1$ na dose mais elevada de cal $(2,0 \%)$. Os teores de $\mathrm{P}, \mathrm{K}, \mathrm{Mg}, \mathrm{Zn}$ e $\mathrm{Cu}$ diminuíram com a elevação da dose de cal $(\mathrm{P}<0,05)$. Em relação ao fator tempo, houve um acréscimo na concentração $\mathrm{K}, \mathrm{Mg}$ e $\mathrm{Mn}(\mathrm{P}<0,05)$. O tratamento da cana-deaçúcar com cal virgem $(\mathrm{CaO})$ microprocessada aumenta o teor de $\mathrm{Ca}$ do volumoso ocasionando um aumento na relação Ca:P. Há presença de $\mathrm{Pb}$ na cal virgem, porém abaixo da concentração tóxica para ruminantes.

Palavras-chave: agente hidrolizante, macro minerais, metais pesados, micro minerais, tratamento alcalino, óxido de cálcio

\section{SUMMARY}

The aim of this study was to evaluate the mineral composition of "in natura" sugarcane hydrolyzed with increasing doses of quicklime and different periods of exposure to air. The levels of quicklime $(\mathrm{CaO})$ used were 0.0 (control); $0.5 ; 1.0 ; 1.5$ to $2.0 \%$ based on natural matter of cane and the time of exposure to air at $0 ; 24 ; 48 ; 72$ and 96 hours. The mineral composition was assessed through the concentrations of calcium $(\mathrm{Ca})$, phosphorus $(\mathrm{P})$, potassium $(\mathrm{K})$, magnesium $(\mathrm{Mg})$, zinc $(\mathrm{Zn})$, copper $(\mathrm{Cu})$, manganese $(\mathrm{Mn})$, chromium $(\mathrm{Cr})$, lead $(\mathrm{Pb})$ and cadmium $(\mathrm{Cd})$. We used a completely randomized design. Data were analyzed using analysis of variance with repeated measures for comparison of means by Tukey test at $5 \%$ probability was used. The Ca concentration changes $(\mathrm{P}<0,05)$ caused a modification of the $\mathrm{Ca}: \mathrm{P}$ ratio in the hydrolyzed sugarcane that reached 36.46:1 at the highest dose of lime (2.0\%). The levels of $\mathrm{P}, \mathrm{K}, \mathrm{Mg}, \mathrm{Zn}$ and $\mathrm{Cu}$ decreased with increasing levels of quicklime $(\mathrm{P}<0,05)$. Regarding to the period of exposure to air, there was an increase in the concentration of $\mathrm{K}, \mathrm{Mg}$, and $\mathrm{Mn}(\mathrm{P}<0,05)$. The treatment of sugarcane with microprocessed quicklime $(\mathrm{CaO})$ increases the $\mathrm{Ca}$ content of the forage. $\mathrm{Pb}$ is present in the quicklime but in a concentration well below the toxic concentration for ruminants.

Keywords: hydrolyzing agent, macro minerals, heavy metals, micro minerals, alkali treatment, calcium oxide 


\section{INTRODUÇÃO}

A tecnologia da hidrólise da cana-deaçúcar com a utilização de óxido ou hidróxido de cálcio consiste do fornecimento da cana-de-açúcar na forma in natura submetida à ação de agentes hidrolíticos imediatamente após a colheita da forragem no campo e posterior armazenamento desse material tratado em ambiente protegido (galpão), possibilitando assim alternativas de manejo que minimizem o corte diário deste volumoso.

Essa técnica vem sendo intensamente pesquisada desde princípios básicos com digestibilidade in vitro $\mathrm{e}$ composição bromatológica (MOTA et al., 2010) a ensaios de desempenho utilizando diferentes espécies e categorias animais. (DOMINGUES et al., 2012; FREITAS et al., 2008; MISSIO et al., 2012) Contudo, raros são os trabalhos que dão destaque à composição mineral, avaliando desde macro e microelementos à concentração de metais pesados da cana-de-açúcar submetida à hidrólise com óxido de cálcio.

Normalmente, os artigos descrevem os valores de cálcio e fósforo como os trabalhos de Romão et al. (2014) e Daniel et al. (2013).

É importante descrever as concentrações de minerais na cana-deaçúcar hidrolisada com cal virgem, pois dependendo do agente alcalinizante utilizado, grandes quantidades de cálcio (Ca) e magnésio $(\mathrm{Mg})$ são acrescentadas à dieta, como também pode ocorrer a presença de metais pesados, visto que a cal virgem é obtida através do processo de calcinação de rochas calcitas e dolomitas e este produto não passa por nenhum processo de purificação onde estes metais pesados poderiam ser removidos.
A inclusão de metais pesados acima dos níveis considerados limite e o desbalanço da relação entre minerais de importância nutricional podem ocasionar distúrbios metabólicos que comprometam a saúde dos animais e, consequentemente, o desempenho.

Neste sentido, objetivou-se avaliar as concentrações de macro, microminerais e metais pesados na cana-de-açúcar hidrolisada com doses crescentes de cal virgem em diferentes tempos de exposição ao ar.

\section{MATERIAL E MÉTODOS}

O experimento foi conduzido na Faculdade de Ciências Agrárias e Veterinárias da Universidade Estadual Paulista (Unesp), Campus de Jaboticabal/SP, na estação seca, no Setor de Bovinocultura de leite, situada a $21^{\circ} 15^{\prime} 22^{\prime}$ ' de latitude sul e $48^{\circ} 18^{\prime}$ $22^{\prime}$ ' $\mathrm{W}$ de longitude oeste, a $610 \mathrm{~m}$ de altitude, com temperatura média anual de $22^{\circ} \mathrm{C}$, pluviosidade $1400 \mathrm{~mm} /$ ano e umidade relativa do ar média de $70,8 \%$.

A cana-de-açúcar utilizada foi a variedade IAC 86-2480, sendo o corte realizado em soqueira com 12 meses $\left(2^{\circ}\right.$ corte), o canavial utilizado no experimento já encontrava-se formado, após o primeiro corte o mesmo foi adubado com $70 \mathrm{~kg}$ de N/ha na forma de uréia, $130 \mathrm{~kg}$ de $\mathrm{P}_{2} \mathrm{O}_{5} /$ ha na forma de superfosfato triplo e $80 \mathrm{~kg} \mathrm{~K}_{2} \mathrm{O}$ na forma de cloreto de potássio. As adubações foram realizadas assim que se iniciou o período de chuvas na região. $\mathrm{O}$ controle de plantas invasoras foi feito através de capina manual.

A cana foi cortada manualmente e picada em picadeira estacionária da marca Menta Mint ${ }^{\circledR}$ para obtenção de partículas entre 8 a $10 \mathrm{~mm}$, em seguida foram feitos amontoados de cana picada 
de aproximadamente $15 \mathrm{~kg}$ (matéria natural) e tratados com doses crescentes de cal virgem $(\mathrm{CaO}), 0,0$ (controle); 0,5; 1,$0 ; 1,5$ e $2,0 \%$ com base na matéria natural da cana, amostras das canas de aproximadamente $300 \mathrm{~g}$ foram retiradas de forma aleatória dos amontoados nos seguintes tempos após o tratamento alcalino $0 ; 24 ; 48 ; 72$ e 96 horas. De acordo com o fabricante, a cal empregada apresentou $90 \%$ de $\mathrm{CaO}$ e $0,5 \%$ de $\mathrm{MgO}$.

Para cada tratamento, a quantidade de óxido de cálcio (cal virgem) utilizada foi diluída em água na quantidade de quatro litros de água para cada $100 \mathrm{~kg}$ de cana-de-açúcar picada com base na matéria natural. Em seguida, as soluções foram distribuídas sobre os amontoados de cana correspondentes e, cuidadosamente, o material foi homogeneizado.

O preparo da solução mineral foi realizado pela "via seca", segundo metodologia descrita por Silva e Queiroz (2002). As leituras dos minerais cálcio $(\mathrm{Ca})$, potássio $(\mathrm{K})$, magnésio $(\mathrm{Mg})$, zinco $(\mathrm{Zn})$, cobre $(\mathrm{Cu})$, manganês $(\mathrm{Mn})$, chumbo $(\mathrm{Pb})$, cádmio (Cd) e cromo (Cr), foram feitas utilizando aparelho de espectofotometria por absorção atômica e o fósforo (P) em aparelho de colorimetria.

Utilizou-se o delineamento inteiramente casualizado, no qual cada unidade experimental era submetida a apenas uma das cinco doses de óxido de cálcio $(0 ; 0,5 ; 1,0 ; 1,5$ e $2,0 \%)$. Para cada tratamento foi estabelecido quatro unidades experimentais, e a cada 24 horas uma amostra era retirada da unidade mensurando os valores dos elementos testados, totalizando assim cinco medidas no tempo $(0,24,48,72$ e 96 horas). Os dados foram analisados usando uma análise de variância
(ANOVA) com medidas repetidas, já que as observações espalhadas no tempo não podem ser consideradas independentes, uma vez que são retiradas de uma mesma unidade experimental. Foi adotado o valor de alfa de $5 \%$ de probabilidade no Teste $\mathrm{F}$ para estimar a significância do teste. Caso a ANOVA apresentasse um resultado significativo para tratamento ou interação, foi usado o teste exato de Tukey para determinar quais médias eram diferentes. Todas as análises foram realizadas com o programa $\mathrm{R}$. ( $\mathrm{R}$ DEVELOPMENT CORE TEAM, 2011).

\section{RESULTADOS E DISCUSSÃO}

A composição mineral da cana-de-açúcar hidrolisada ou não com $\mathrm{CaO}$ encontra-se na Tabela 1.

Não foram encontrados cádmio e cromo nas amostras analisadas independentemente do tratamento, podendo-se inferir que nem a cana-deaçúcar nem a cal possuem concentrações representativas destes minerais. De fato, Valadares Filho et al. (2008), em ampla compilação de resultados de análises bromatológicas de alimentos para bovinos, não citam a presença destes minerais na cana-de-açúcar.

Não houve interação entre dose de cal e tempos de exposição ao ar para nenhuma das variáveis avaliadas $(\mathrm{P}>0,05)$.

As maiores concentrações de $\mathrm{Ca}$ encontradas foram para as doses 1,5 e $2,0 \%$ de cal virgem, isto porque o agente alcalinizante utilizado (cal virgem) continha $94,1 \%$ de $\mathrm{CaO}$, o que promoveu um aumento nos teores de $\mathrm{Ca}$ à medida em que se aumentava a dose de $\mathrm{CaO}$. Não houve efeito do tempo de exposição ao ar para as concentrações de $\mathrm{Ca}(\mathrm{P}>0,05)$. 
Tabela 1. Médias de cálcio $(\mathrm{Ca})$, fósforo $(\mathrm{P})$, potássio $(\mathrm{K})$, magnésio $(\mathrm{Mg})$, zinco $(\mathrm{Zn})$, cobre $(\mathrm{Cu})$, manganês $(\mathrm{Mn})$ e chumbo $(\mathrm{Pb})$ da cana-de-açúcar hidrolisada com doses crescentes de cal virgem $(\mathrm{CaO})$ microprocessada e diferentes tempos de exposição ao ar

\begin{tabular}{|c|c|c|c|c|c|c|c|c|c|c|c|c|c|c|c|}
\hline \multirow{2}{*}{ Par. } & \multicolumn{5}{|c|}{ Dose de cal (\%) } & \multicolumn{5}{|c|}{ Tempo de exposição ao ar (horas) } & \multicolumn{3}{|c|}{ Efeito $^{1}$} & \multirow{2}{*}{$\begin{array}{l}\mathrm{CV}^{2} \\
(\%)\end{array}$} & \multirow{2}{*}{$\begin{array}{l}\mathrm{CV}^{3} \\
(\%)\end{array}$} \\
\hline & 0,0 & 0,5 & 1,0 & 1,5 & 2,0 & 0 & 24 & 48 & 72 & 96 & $\mathrm{D}$ & $\mathrm{T}$ & DxT & & \\
\hline $\mathrm{Ca}$ & $0,23^{\mathrm{D}}$ & $1,25^{\mathrm{C}}$ & $2,19^{\mathrm{B}}$ & $3,10^{\mathrm{A}}$ & $3,06^{\mathrm{A}}$ & 1,70 & 1,92 & 2,02 & 2,04 & 2,15 & $*$ & NS & NS & 15,7 & 14,2 \\
\hline $\mathrm{P}$ & $0,12^{\mathrm{A}}$ & $0,12^{\mathrm{A}}$ & $0,12^{\mathrm{A}}$ & $0,10^{\mathrm{B}}$ & $0,08^{\mathrm{C}}$ & 0,11 & 0,10 & 0,11 & 0,11 & 0,11 & * & NS & NS & 18,2 & 16,9 \\
\hline $\mathrm{K}$ & $0,88^{\mathrm{A}}$ & $0,75^{\mathrm{B}}$ & $0,74^{\mathrm{BC}}$ & $0,70^{\mathrm{BC}}$ & $0,64^{\mathrm{C}}$ & $0,67^{\mathrm{c}}$ & $0,68^{\mathrm{c}}$ & $0,75^{\mathrm{b}}$ & $0,77^{\mathrm{b}}$ & $0,83^{\mathrm{a}}$ & $*$ & $*$ & NS & 15,4 & 5,9 \\
\hline $\mathrm{Mg}$ & $0,11^{\mathrm{A}}$ & $0,11^{\mathrm{A}}$ & $0,09^{\mathrm{C}}$ & $0,11^{\mathrm{A}}$ & $0,10^{\mathrm{B}}$ & $0,10^{\mathrm{b}}$ & $0,10^{\mathrm{b}}$ & $0,10^{\mathrm{b}}$ & $0,11^{\mathrm{a}}$ & $0,11^{\mathrm{a}}$ & $*$ & $*$ & NS & 10,6 & 8,4 \\
\hline $\mathrm{Zn}$ & $22,71^{\mathrm{A}}$ & $18,83^{\mathrm{AB}}$ & $13,45^{\mathrm{B}}$ & $16,92^{\mathrm{AB}}$ & $13,05^{\mathrm{B}}$ & 14,25 & 13,80 & 19,25 & 17,45 & 20,15 & $*$ & NS & NS & 50,9 & 50,8 \\
\hline $\mathrm{Cu}$ & $3,55^{\mathrm{A}}$ & $2,15^{\mathrm{B}}$ & $1,90^{\mathrm{B}}$ & $2,45^{\mathrm{B}}$ & $1,85^{\mathrm{B}}$ & 3,15 & 2,25 & 2,60 & 1,95 & 1,95 & $*$ & NS & NS & 44,5 & 42,3 \\
\hline $\mathrm{Mn}$ & 31,70 & 33,45 & 31,75 & 33,80 & 34,00 & $29,70^{\mathrm{b}}$ & $30,85^{\mathrm{b}}$ & $35,40^{\mathrm{a}}$ & $33,55^{\mathrm{a}}$ & $35,2^{\mathrm{a}}$ & NS & $*$ & $\mathrm{NS}$ & 13,1 & 9,1 \\
\hline $\mathrm{Pb}$ & $0,00^{\mathrm{B}}$ & $1,15^{\mathrm{B}}$ & $4,30^{\mathrm{A}}$ & $5,6^{\mathrm{A}}$ & $6,25^{\mathrm{A}}$ & 4,85 & 3,30 & 3,80 & 2,50 & 2,85 & $*$ & NS & NS & 85,2 & 86,1 \\
\hline
\end{tabular}

Médias seguidas de letras diferentes maiúsculas em relação à dose de cal e minúsculas em relação ao tempo de exposição ao ar, diferem estatisticamente entre si pelo teste Tukey $\left({ }^{*} \mathrm{P}<0,05\right)$. Os teores de $\mathrm{Ca}, \mathrm{P}, \mathrm{K}$ e $\mathrm{Mg}$ estão expressos em \%MS e os teores de $\mathrm{Zn}, \mathrm{Cu}, \mathrm{Mn}$, e $\mathrm{Pb}$ estão expressos em mg/kg. ${ }^{\mathrm{I}} \mathrm{Probabilidades} \mathrm{de} \mathrm{P}<\mathrm{F}$, sendo D: doses de cal, T: tempo de exposição ao ar e DxT interação entre dose e tempo. ${ }^{*} \mathrm{P}<0,05$. NS = não significativo. ${ }^{2}$ coeficiente de variação da dose de cal, ${ }^{3}$ coeficiente de variação do tempo de exposição ao ar. 
A menor concentração de $\mathrm{P}$ foi encontrada para a dose de $2,0 \%$ de cal virgem $(\mathrm{P}<0,05)$ e não houve efeito do tempo para as concentrações desse mineral.

De acordo com Suttle et al. (1991), bovinos apresentam grande tolerância à ingestão de $\mathrm{Ca}$ em excesso às suas necessidades, desde que os requisitos de $\mathrm{P}$ estejam atendidos. No entanto, relações $\mathrm{Ca}: \mathrm{P}$ superiores a $8: 1$ podem comprometer o desempenho animal.

Neste estudo, a relação $\mathrm{Ca}: \mathrm{P}$ teve grande variação, para a cana sem cal $(0,0 \%)$, a relação $\mathrm{Ca}: \mathrm{P}$ foi de $1,85: 1$, esta relação é muito próxima da ideal (2:1); no entanto, os teores destes minerais ( $\mathrm{Ca}$ e P) estão abaixo das exigências nutricionais das diversas categorias dos animais ruminantes. Ao adicionar quantidades crescentes de cal à cana, observou-se também um aumento na relação $\mathrm{Ca}: \mathrm{P}$ cujas relações foram de 10,70:1; 18,07:1; 30,65:1; $36,46: 1$; para as doses 0,$5 ; 1,0 ; 1,5 \mathrm{e}$ $2,0 \%$, respectivamente. Estes valores são extremamente elevados, não sendo possível o fornecimento exclusivo da cana-de-açúcar hidrolisada para animais ruminantes.

Deve-se ressaltar que os cálculos aqui apresentados para a relação $\mathrm{Ca}: \mathrm{P}$ estão levando em consideração os percentuais brutos de cada mineral na cana-deaçúcar hidrolisada, isto é, sem as suas respectivas biodisponibilidades, o que pode diminuir esta relação. Segundo Coelho da Silva (1995), a quantidade de $\mathrm{Ca}$ e $\mathrm{P}$ absorvida no trato digestivo é função da disponibilidade destes elementos no alimento, dos requerimentos e quantidades ingeridas pelo animal e da relação Ca:P. À medida que o $\mathrm{Ca}$ dietético aumenta acima dos requerimentos do animal, a absorção de ambos os elementos é reduzida, visto que esses nutrientes inorgânicos estão intimamente relacionados (COELHO DA SILVA, 1995).

Houve efeito da dose cal $(\mathrm{P}<0,05)$ para as concentrações de $\mathrm{K}, \mathrm{Mg}, \mathrm{Zn}$ e $\mathrm{Cu}$. As maiores concentrações de $\mathrm{K}$ e $\mathrm{Cu}$ foram encontradas no tratamento sem cal, os menores valores descritos para os tratamentos com cal, ocorreram devido a menor participação centesimal destes minerais na fração mineral do alimento tratado. Com a inclusão da cal virgem, ocorre um aumento na quantidade de $\mathrm{Ca}$ na cana, a quantidade de $\mathrm{K}$ e $\mathrm{Cu}$ não sofrem alteração, porém $\mathrm{O}$ seu percentual diminui em relação ao total de minerais.

A menor concentração de $\mathrm{Mg}$ foi encontrada na dose de $1,0 \%$, este resultado não era o esperado, haja visto que a cal utilizada apresenta, segundo o fabricante, teor de $\mathrm{MgO}$ de $0,5 \%$, o que levaria ao aumento nas concentrações de $\mathrm{Mg}$ com o aumento da dose de cal virgem microprocessada. Este fato demonstra que teor de $\mathrm{MgO}$ na cal virgem pode ser muito variável e menor do que o descrito pelo fabricante e ainda por se tratar de uma rocha, a quantidade de $\mathrm{MgO}$ pode sofrer grandes variações. Mota et al. (2010), estudando a cana-deaçúcar hidrolisada com cal virgem e cal hidratada, não observaram aumento no teor de $\mathrm{Mg}$ da cana-de-açúcar hidrolisada.

As maiores concentrações de $\mathrm{Zn}$ foram descritas para as doses $0,0,0,5$ e 1,5\%, sendo que houve diferença significativa apenas entre as doses 0,0 e 2,0\%.

Não houve efeito da dose de $\mathrm{CaO}$ para os teores de $\mathrm{Mn} \quad(\mathrm{P}>0,05)$. As concentrações de $\mathrm{Ca}, \mathrm{P}, \mathrm{Zn}, \mathrm{Cu}$ e $\mathrm{Pb}$ não sofreram efeito do tempo de exposição ao ar $(\mathrm{P}>0,05)$. Resultados similares foram descritos por Mota et al. (2010) para as concentrações de Ca e P com o tempo de exposição ao ar variando de 12 a 60 horas. 
Houve efeito do tempo de exposição ao ar sobre os teores de $\mathrm{K}, \mathrm{Mg}$ e $\mathrm{Mn}$ $(\mathrm{P}<0,05)$. A maior concentração de $\mathrm{K}$ foi encontrada no maior tempo de exposição ao ar. Em relação ao $\mathrm{Mg}$, os resultados dos tempos 72 e 96 horas foram semelhantes entre si e superiores que os demais. As menores concentrações de Mn foram encontradas nos tempos 0 e 24 horas. De modo geral, observa-se que há um tendência de elevação nas concentrações de $\mathrm{K}$, $\mathrm{Mg}$ e Mn com o aumento do tempo de exposição ao ar.

De acordo com Domingues et al. (2011), com o passar das horas da cana-de-açúcar exposta ao ar, ocorre o desenvolvimento de microorganismos, principalmente leveduras, que consomem os carboidratos solúveis presente na cana. $\mathrm{O}$ consumo destes carboidratos fazem com que as concentrações dos demais nutrientes aumentem percentualmente.

Além da variação no teor dos macro e microminerais, existe a possibilidade da presença de metais pesados nas cales. Malavolta (1994) relatou teores variando de 5 a $100 \mathrm{mg} / \mathrm{kg}$ de $\mathrm{Pb}$ em calcários de acordo com a literatura internacional e 23 a $28 \mathrm{mg} / \mathrm{kg}$ para calcários produzidos em Minas Gerais, Brasil. A afirmação de Malavolta (1994), indica que pode ocorrer a presença de $\mathrm{Pb}$ na cal, haja visto que esta é produzida a partir da calcinação ou queima do calcário.

Com exceção da dose $0,5 \%$, todas as demais doses apresentaram concentrações $\mathrm{de} \mathrm{Pb}$ superiores ao tratamento controle, constatando-se a presença deste mineral na cal, haja visto que não foi encontrado $\mathrm{Pb}$ no tratamento sem cal. No entanto, os valores de $\mathrm{Pb}$ encontrados são inferiores às concentrações necessárias para causar intoxicação para ruminantes, que segundo o National Research Council (NRC, 1996) é de 30 ppm.
$\mathrm{O} \mathrm{Pb}$ apresenta características de toxicidade aguda e crônica, pelo seu efeito acumulativo nos órgãos dos animais e consequentemente do ser humano (GONÇALVES et al., 2008). Contudo, o conceito sobre minerais tóxicos deve ser feito de maneira cuidadosa e isto é conveniente pelo fato de alguns minerais serem considerados essenciais, mas em concentrações muito elevadas pode causar intoxicação aos animais ou de maneira indireta causar a deficiência de um segundo mineral devido ao antagonismo entre eles. (MENDONÇA JUNIOR et al., 2011).

$\mathrm{O}$ tratamento da cana-de-açúcar com cal virgem microprocessada $(\mathrm{CaO})$ altera a composição mineral da cana-de-açúcar, principalmente, causando elevação nas concentrações $\mathrm{Ca}$ e consequentemente aumentando a relação Ca:P. Há presença de $\mathrm{Pb}$ na cal virgem, porém, abaixo da concentração tóxica para ruminantes.

\section{REFERÊNCIAS}

COELHO DA SILVA, J.F. Exigências de macroelementos inorgânicos para bovinos: o sistema ARC/AFRC e a experiência no Brasil. In: Simpósio internacional sobre exigências nutricionais de ruminantes, Anais... Viçosa, MG: Universidade Federal de Viçosa, 1995. p.467-503.

DANIEL, J.L.P.; SANTOS, M.C.; ZOPOLLATTO, M.; HUHTANEN, P.; NUSSIO, L.G. A data-analysis of lime addition on the nutritive value of sugarcane in Brazil. Animal Feed Science and Technology, v.184, p.1723, 2013. 
Rev. Bras. Saúde Prod. Anim., Salvador, v.15, n.4, p.827-834 out./dez., 2014 http://www.rbspa.ufba.br ISSN 15199940

DOMINGUES, F.N.; OLIVEIRA, M.D.S.; SIQUEIRA G.R.; ROTH, A.P.T.P., ;SANTOS, J.; MOTA, D.A. Estabilidade aeróbia, $\mathrm{pH}$ e dinâmica de desenvolvimento de microorganismos da cana-de-açúcar in natura hidrolisada com cal virgem. Revista Brasileira de Zootecnia, v.40, p.715-719, 2011.

DOMINGUES, F.N.; OLIVEIRA, M.D.S.; MOTA, D.A.; FERREIRA, D.S.; SANTOS, J. Desempenho de novilhas de corte alimentadas com cana hidrolisada. Ciência Animal

Brasileira, v.13, p.8-14, 2012.

FREITAS, A.W.P.; ROCHA, F.C.; ZONTA, A. FAGUNDES, J.L., FONSECA, R., ZONTA, M.C.M., MACEDO, F.L. Consumo de nutrientes e desempenho de ovinos alimentados com dietas à base de cana-de-açúcar hidrolisada. Pesquisa Agropecuária Brasileira, v.43, p.1569-1574, 2008.

GONÇALVES, J.R.; MESQUITA, A.J.; GONÇALVES, R.M. Determinação de metais pesados em leite integral bovino pasteurizado no estado de Goiás.

Ciência Animal Brasileira, v.9, p.365374, 2008.

MALAVOLTA, E. Fertilizantes e seu impacto ambiental: mitos, mistificações e fatos. São Paulo: Produção Química, 1994. 153p.

MENDONÇA JÚNIOR, A.F.; BRAGA, A.P.; RODRIGUES, A.P.M.S.; SALES, L.E.M.; MESQUITA, H.C. Minerais: importância de uso na dieta de ruminantes. ACSA - Agropecuária Científica no Semi-árido, v.7, p.1-13, 2011.

MISSIO, R.L.; OLIVEIRA, M.D.S.; SFORCINI, M.P.R.; RENNÓ, F.P.; FREITAS JÚNIOR, J.E.; ELEJADE, D.A.G.; FERRARI, V.B.; ABUD, G.C.
Digestion of feed fractions and intake of heifers fed hydrolyzed sugarcane stored for different periods. Revista Brasileira de Zootecnia, v.41, p.1737-1746, 2012.

MOTA, D.A.; OLIVEIRA, M.D.S.; DOMINGUES, F.N.; MANZI, G.M.; FERREIRA, D.S.; SANTOS, J. Hidrólise da cana-de-açúcar com cal virgem ou cal hidratada. Revista Brasileira de Zootecnia, v.39, p.11861190, 2010.

NATIONAL RESEARCH COUNCIL NRC. Nutrients requeriments of beef cattle. Washington, D.C.: National Academy Press, 1996. 244p.

NATIONAL RESEARCH COUNCIL NRC. Nutrients requeriments of beef cattle. Washington, D.C.: National Academy Press, 2001. 381p.

R DEVELOPMENT CORE TEAM, R. $R$ : a language and environment for statistical ComputingR Foundation for Statistical Computing., 2011. Disponível em: $<\mathrm{http}: / / w w w . r-$ project.org>. Acesso em: 29 nov. 2014.

ROMÃO, C.O.; CARVALHO, G.G.P.; LEITE, V.M.; SANTOS, A.S.; CHAGAS, D.M.T.; RIBEIRO, O.L.; OLIVEIRA, P.A.; MAGALHÃES, A.F.; PIRES, A.J.V. Chemical composition and dry matter digestibility of sugar cane oxide treat with calcium. Arquivo Brasileiro de Medicina Veterinária e Zootecnia, v.66, p.529538, 2014.

SILVA, D.J.; QUEIROZ, A.C. Análise de alimentos: métodos químicos e biológicos. Viçosa, MG: Universidade Federal de Viçosa, 2002. 235p. 
SUTTLE, N.F.; ARMSTRONG, D.G.; BRAITHWAITE, G.D.; FIELD, A.C., SCOTT, D.; THOMPSON, J.K.;

WOOLLIAMS, J. A reappraisal of the calcium and phosphorus requirements of sheep and cattle. IN: Agricultural and Food Research Council: Technical Committee on Responses to Nutrients Report. Nutrition Abstracts and Reviews, v.61, p.573-612, 1991.

VALADARES FILHO, S.C., MACHADO, P.A.S., CHIZZOTTI, M.L., AMARAL, H.F., MAGALHÃES, K.A., ROCHA JUNIOR, V.R., CAPELLE, E.R. Tabelas brasileiras de composição de alimentos para bovinos. CQBAL 3.0. Viçosa, MG: Universidade Federal de Viçosa, 2008. $502 p$.

Data de recebimento: 17/02/2014

Data de aprovação: 13/10/2014 